\title{
Effect of Fetal Choroid Plexus Cysts on Middle Cerebral Artery Doppler Parameters
}

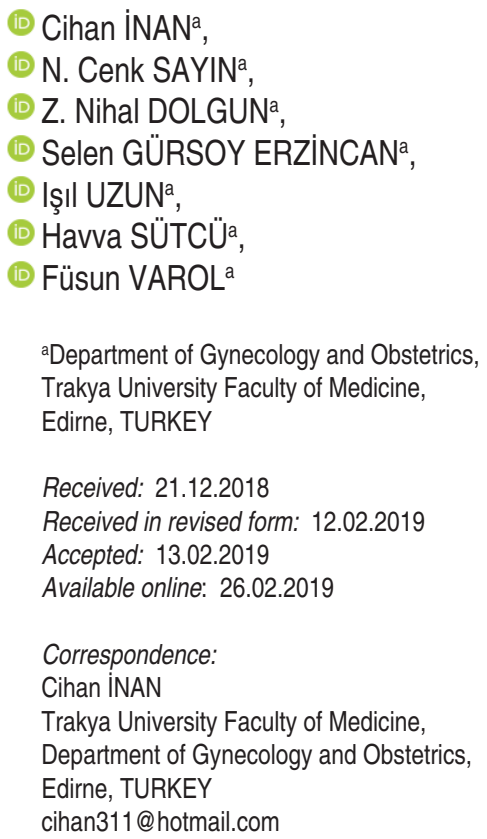

Copyright $@ 2019$ by Türkiye Klinikleri

\begin{abstract}
Objective: To investigate the possible effects of isolated fetal choroid plexus cysts (CPCs) on middle cerebral artery (MCA) Doppler parameters. Material and Methods: A total of 113 pregnant women at 18 to 22 gestational weeks in whom level 2 ultrasonography (USG) was performed between August 2017 and January 2018 were included in the study. Fifty-five women (CPC group) had isolated fetal CPC, whereas 58 women (control group) had no fetal structural anomaly. We measured the following MCA Doppler parameters of fetuses: peak systolic velocity (PSV), end-diastolic velocity (EDV), the systolic/diastolic ratio (S/D), pulsatility index (PI), and resistance index (RI). We examined whether a significant difference exist between the CPC and control groups, and between the CPC and contralateral sides with respect to the Doppler parameters. Results: We found that cysts were present in the right lateral ventricle in $49.1 \%$ ( $n=27)$ individuals in the CPC group. There was no statistically significant difference between the groups in terms of PSV, EDV, PI and RI values; however, the S/D ratio exhibited statistical significance. The MCA S/D ratio of the control group was lower than that of the values reported for both cystic and contralateral sides in the CPC group. Moreover, we did not observe any significant difference in S/D ratios between the cystic and contralateral sides in the CPC group. Conclusion: Isolated fetal CPCs tend to occur more on the right side unilaterally. At 18 to 22 weeks of gestation, MCA $\mathrm{S} / \mathrm{D}$ ratios of the cystic and contralateral sides in the CPC group were higher than that in the control group.
\end{abstract}

Keywords: Choroid plexus cyst; middle cerebral artery; Doppler ultrasonography; fetus

horoid plexuses are highly vascularized secretory tissues that produce cerebrospinal fluid (CSF) in the brain. Its development starts in the lateral ventricles at the sixth gestational week and by the ninth gestational week, it acquires the ability to produce CSF. The anterior and posterior choroidal arteries supply blood to the choroid plexus and its anterior branch is derived from the middle cerebral artery (MCA). ${ }^{1}$ Choroid plexus cysts (CPCs) are fluid-filled structures that are formed in the fetus when CSF gets trapped in the intervillous clefts of the rapidly growing choroid plexuses. ${ }^{2,3}$ These cysts are visible as lesions in the routine secondtrimester obstetric ultrasonography (USG) with a frequency of $0.5 \%$ to $3.6 \%{ }^{4}$ These can be unilateral or bilateral and single or multiple. ${ }^{5}$ In an obstetric USG, CPCs are seen in an axial plane in the atrial portions of the lateral ventricle, generally at the level of the glomus. The real CPCs appear as round sonolucent structures with internal septations and may protrude into the ventricular cavity. ${ }^{5,6}$ 
Choroid plexus cysts are generally benign malformations and tend to resolve by the 25th to 28th gestational week. In rare cases, these may coexist with fetal aneuploidy, particularly trisomy 18 . In routine obstetric practices, prenatal invasive procedure is recommended when it coexists with an additional soft marker. ${ }^{6}$ If CPCs do not resolve, these may obstruct the flow of spinal fluid, thereby causing ventriculomegaly by creating a mass effect with a shift to the foramen of Monro. In the neonatal period, this may cause neurological symptoms by increasing the intracranial pressure. ${ }^{7}$

The present study investigated whether CPCs, which are generally considered benign and temporary lesions, could affect MCA Doppler parameters by creating a possible mass effect in the second trimester.

\section{MATERIAL AND METHODS}

The present prospective study was conducted between August 2017 and January 2018 at the Faculty of Medicine, Division of Perinatology, Trakya University. The ethical consent was obtained from the Ethics Committee of Trakya University (Approval number: 2017/306, decision number: 20/23, 2017). The study was conducted in accordance with the Helsinki declaration. Written consents were received from all patients before the study.

Our study included 113 pregnant women at 18 to 22 gestational weeks who presented to our clinic between the specified duration. Fifty-five of them had isolated fetal CPC (CPC group) and 58 did not have CPC (control group). Gestational age was determined according to the last menstrual period corrected with first-trimester USG measurements. CPC group did not report any additional fetal pathology, and the control group did not have any structural anomaly. The exclusion criteria included having additional structural fetal anomalies along with CPC, having other soft markers, multiple pregnancies, fetal chromosomal anomalies, oligopolyhydramnios, fetal growth retardation, perinatal infections, using drugs that affect vascular tone (such as antihypertensive drugs), and maternal chronic diseases.
Fetal measurements and the MCA Doppler index measurements were performed with the Voluson E6 Expert (General Electric; Zipf, Austria). The USG examinations were performed by a single investigator (C.I.). For Doppler measurements, the insonation angle was held at $<30$ degrees with a sample volume at $2 \mathrm{~mm}$. The right and left MCA Doppler measurements were taken from the distal portion of the vessel that was $1 \mathrm{~cm}$ away from its origin from the circle of Willis. The Doppler parameters including peak systolic velocity (PSV), end-diastolic velocity (EDV), systolic/diastolic $(\mathrm{S} / \mathrm{D})$ ratio, pulsatility index $(\mathrm{PI})$, and resistance index (RI) were calculated automatically with USG. The size of CPCs was measured at the widest axis.

First, size and location (side) of CPCs were recorded. The MCA Doppler parameters of the CPC and contralateral sides were then measured. Similarly, the MCA Doppler parameters were measured for both sides in the control group. We next examined whether there existed a difference between the Doppler parameters of the two sides in the control group. We also investigated whether a significant difference existed in Doppler parameters between the CPC side and contralateral side in the CPC group and also between these parameters in the CPC and control groups.

\section{STATISTICAL ANALYSIS}

The SPSS version 22.0 (IBM Corp; Armonk, NY, United States) was used for statistical analysis. The data were evaluated using descriptive statistical methods (mean, standard deviation, standard error, median, first and third quartiles, frequency, percentage, minimum, and maximum). The conformity of quantitative variables to a normal distribution was tested using the Shapiro-Wilk test and by graphical evaluation. An independent groups $t$-test was used for evaluating the normally distributed variables between the two independent groups. The Mann-Whitney $U$ test was used for evaluating the non-normally distributed variables between the two independent groups. Dependent groups $t$-test was used for evaluating normally distributed variables between the right and left sides. Generalized linear mixed models were used for 


\begin{tabular}{|c|c|c|c|}
\hline & $\mathrm{CPC}$ group & Control group & $p$ \\
\hline Maternal age (years; mean \pm s.d.) & $28.43 \pm 6.45$ & $29.67 \pm 0.87$ & ${ }^{\mathrm{a}} 0.272$ \\
\hline${ }^{\ddagger}$ Gravidity (n) & $2(1-3)$ & $2(1-3)$ & ${ }^{\mathrm{b}} 0.728$ \\
\hline 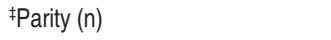 & $1(0-2)$ & $1(0-2)$ & ${ }^{\mathrm{b}} 0.869$ \\
\hline \multirow[t]{2}{*}{${ }^{\ddagger}$ Gestational age (weeks) } & $19.57(18.86-21)$ & $20(19-21)$ & ${ }^{\mathrm{b}} 0.179$ \\
\hline & $n(\%)$ & $n(\%)$ & \\
\hline \multicolumn{4}{|l|}{ Fetal gender; $\mathrm{n}(\%)$} \\
\hline Male & $26(47.3)$ & $28(48.3)$ & ${ }^{0} 0.915$ \\
\hline Female & $29(52.7)$ & $30(51.7)$ & \\
\hline
\end{tabular}

aIndependent samples t-test

'Mann-Whitney U test

'Pearson's chi-square test

$\ddagger$ Values are reported as median (first quartile, third quartile).

${ }^{* *} \mathrm{p}<0.01$

CPC: choroid plexus cysts, s.d.: Standard deviation.

comparing PSV, EDV, S/D, and PI and RI values of study-healthy, study-patient, and control group cases. Pearson's chi-square test and one-sample chisquare test were utilized to compare the qualitative data. The statistical significance was set at $\mathrm{p}<0.05$.

\section{RESULTS}

We did not find any statistically significant difference between the CPC and control groups with respect to maternal age, gravidity and parity values, and gestational age in the USG examination ( $>>0.05)$. Moreover, no significant difference between the groups was observed in terms of fetal gender $(p>0.05)$. Table 1 indicates the demographic features of the groups.

In the CPC group, the cyst size varied between $2.7 \mathrm{~mm}$ and $13.6 \mathrm{~mm}$ (median: $5.7 \mathrm{~mm}$ ); the mean size was $6.2 \pm 2.7 \mathrm{~mm}$. With respect to location, cysts were present in the right lateral ventricle in $49.1 \%$ ( $\mathrm{n}=27$ ), in left lateral ventricle in $21.8 \%$ $(\mathrm{n}=12)$, and bilateral in $29.1 \%(\mathrm{n}=16)$ of cases. Thus, a majority of individuals had right side cysts $(\mathrm{p}=$ 0.037; Table 2).

There was no statistically significant difference in the control group in terms of right and left MCA PSV, EDV, S/D, and PI and RI values ( $p>0.05$; Table 3). Five different models were used to investigate the effect of group variable on PS, ED, S/D, and PI
TABLE 2: Distribution of choroid plexus cysts according to their locations.

\begin{tabular}{|lcccc|}
\hline \multicolumn{1}{l}{} & $\mathrm{n}$ & $\%$ & Expected $\mathrm{n}$ & Residual \\
Cyst side & & & & \\
$\quad$ Right & 27 & 49.1 & 18.333 & 8.666 \\
Left & 12 & 21.8 & 18.333 & -6.333 \\
$\quad$ Right-left & 16 & 29.1 & 18.333 & -2.333 \\
Total & 55 & 100.0 & & \\
\hline
\end{tabular}

One sample chi-square test $p=0.037$.

TABLE 3: Comparison of the right and left side middle cerebral artery Doppler parameters in the control group.

\begin{tabular}{|lccc|}
\hline & Right & Left & \\
Control & Mean \pm s.d. & Mean \pm s.d. & p \\
\hline PSV & $23.16 \pm 4.49$ & $23.15 \pm 3.97$ & ${ }^{\circ} 0.984$ \\
\hline EDV & $5.97 \pm 1.42$ & $5.99 \pm 1.68$ & ${ }^{\circ} 0.917$ \\
S/D & $3.90 \pm 0.74$ & $4.11 \pm 1.35$ & ${ }^{\circ} 0.128$ \\
PI & $1.45 \pm 0.21$ & $1.49 \pm 0.29$ & ${ }^{\circ} 0.295$ \\
RI & $0.74 \pm 0.06$ & $0.74 \pm 0.07$ & ${ }^{\circ} 0.692$ \\
\hline
\end{tabular}

PSV: Peak systolic velocity; EDV: End-diastolic velocity; S/D: Systolic/diastolic ratio, PI: Pulsatility index; RI: Resistance index; s.d.: Standard deviation.

${ }^{\mathrm{d} P a i r e d}$ samples t-test.

and RI values. Generalized linear mixed models were used considering that the data of cystic and contralateral sides in the CPC group were dependent and that the CPC group and control group data 


\begin{tabular}{|c|c|c|c|c|c|}
\hline & $\begin{array}{c}\text { CPC group } \\
\text { Contralateral side MCA } \\
\text { Mean (s.e.) }\end{array}$ & $\begin{array}{c}\text { CPC group } \\
\text { Cyst side MCA } \\
\text { Mean (s.e.) }\end{array}$ & $\begin{array}{c}\text { Control group } \\
\text { Mean (s.e.) }\end{array}$ & $F$ & $p$ \\
\hline PSV & $23.64(0.76)$ & $23.97(0.63)$ & $23.16(0.65)$ & 0.409 & 0.665 \\
\hline EDV & $5.54(0.29)$ & $5.77(0.24)$ & $5.97(0.25)$ & 0.668 & 0.514 \\
\hline$S / D$ & $4.56(0.20)$ & $4.55(0.17)$ & $3.90(0.17)$ & 3.973 & $0.021^{*}$ \\
\hline PI & $1.51(0.04)$ & $1.52(0.03)$ & $1.45(0.04)$ & 1.143 & 0.321 \\
\hline RI & $0.76(0.01)$ & $0.76(0.01)$ & $0.74(0.01)$ & 1.913 & 0.151 \\
\hline
\end{tabular}

CPC: Choroid plexus cysts; MCA: Middle cerebral artery; PSV: Peak systolic velocity; EDV: End-diastolic velocity; S/D: Systolic/diastolic ratio; PI: Pulsatility index; RI: Resistance index; s.e.: Standard error.

${ }^{*} \mathrm{p}<0.05$; F: Test value.

were independent of each other. Since there was no statistically significant difference between the right- and left-side values in the control group, we believed that using the data of any side from the control group would not create bias when comparing with the CPC group. In this context, the rightside data of the control group were included as the control group data in the models. Group variable was included as an independent factor, whereas PS, ED, S/D, PI, and RI values were included as dependent variables in the models. We did not find a statistically significant effect of the group variable on PSV, EDV, and PI and RI values ( $p>0.05)$. However, the effect of the group variable on the $\mathrm{S} / \mathrm{D}$ ratio was statistically significant ( $\mathrm{p}=0.021$; Table 4$)$. Bonferroni-corrected post-hoc assessments indicated that the $S / D$ value of the control group was lower than that of both the cystic and contralateral side values of the CPC group ( $\mathrm{p}=0.029$ and $\mathrm{p}=0.026$, respectively). No significant difference in $\mathrm{S} / \mathrm{D}$ ratios between the cystic and contralateral sides of the CPC group was reported $(\mathrm{p}=0.937)$.

\section{DISCUSSION}

The present study evaluated the possible effects of isolated fetal CPCs on MCA Doppler parameters. It was observed that fetal CPC cases exhibited higher MCA S/D ratios as compared to the control group. The MCA S/D values of both sides were similar in the CPC group. No significant difference existed between the control and CPC groups in terms of other Doppler parameters.
The field of fetal CPCs has largely remained unexplored. Fetal CPCs are generally temporary and benign lesions, with no major clinical implications, as evident from the lack of pathophysiological studies in this area. The isolated CPCs are considered as normal variants. Moreover, $90 \%$ of CPCs resolve spontaneously by the $28^{\text {th }}$ week of gestation; so these rarely persist postnatally. It is believed that a CPC, by itself, does not cause neurological damage, or cognitive and motor behavioral damage. Only a few studies in neurosurgery literature have reported large CPCs to cause postnatal intracranial hypertension. ${ }^{8}$ Furthermore, cysts can occur at equal rates in both male and female fetuses; their real incidence depends on the resolution of the USG device and the attention of the operator. ${ }^{8}$ In the present study, we found isolated CPCs to occur at equal rates in both the genders, an observation consistent with that reported in the literature.

Numerous studies have been conducted on CPCs. A study reported the mean gestational age for detecting CPC to be $19.0 \pm 2.1$ weeks and $56.7 \%$ of cases to have unilateral CPCs. ${ }^{9}$ Another study reported the mean gestational age for detecting fetal CPCs to be $19.2 \pm 1.5$ weeks. ${ }^{10}$ In our study, the median gestational week at which fetal CPCs were detected was 19.57 weeks, which was consistent with that reported in the literature. The cysts were unilateral in approximately $70.9 \%$ of our cases, with the incidence of cysts on the right side to be significantly higher. A few studies in the literature ex- 
amined the unilateral or bilateral occurrence of cysts as well. ${ }^{9,11,12}$ However, no study exists that reports a more common side for cyst occurrence. We believe that more extensive studies are required to decipher the mechanism behind the common occurrence of cyst on one side. In addition, a study reported the mean size of cysts to be $6.08 \pm 3.0 \mathrm{~mm} .^{9}$ The mean cyst size observed in the present study was in coherent with that reported in the literature.

Fetal CPC cases having additional anomalies or abnormal screening test results are associated with genetic anomalies such as trisomy $18 .{ }^{13}$ The literature available on this aspect reports that CPC may coexist with congenital cardiac anomalies and hydronephrosis. Therefore, cases with CPC should be carefully investigated for any additional findings. ${ }^{14}$ None of the cases in the present study reported any additional structural fetal anomaly.

Since most CPCs resolve spontaneously, regular follow-ups with repeated USG examinations are not required. ${ }^{9}$ In very few cases, these may cause obstructive hydrocephaly in the postnatal period. ${ }^{15}$ Also, cysts may be rarely symptomatic. ${ }^{16}$ Another finding in the literature is that CPCs could be flexible and may float, thereby changing their location to the foramen of Monro and obstructing CSF pathways. ${ }^{17}$ For instance, a study reported isolated CPC cases to be associated with ventriculomegaly, resulting due to physical distention of large CPCs or the obstruction of the foramen of Monro. ${ }^{7}$ Our aim in the present study was to identify whether these cysts caused a mass effect by affecting the MCA Doppler parameters. For this, we focused on MCA Doppler parameters owing to the contribution of MCA to the blood supply to the choroid plexus. Another reason for its selection was its preference for evaluating the fetal cerebral circulation. ${ }^{1,18}$

Intracranial pressure is affected by the presence of intracranial masses. Initially, the pressure increases in the craniospinal cavity due to the mass effect. The increased intracranial pressure affects the cerebral hemodynamics and cerebral perfusion. According to the Starling hypothesis, increased intracranial pressure causes elevation of cerebral venous pressure, which is attributed to reduced cerebral blood flow. ${ }^{19}$ Nontumoral intracranial cystic masses may also exert a mass effect on the brain..$^{20}$ For example, a study examined the cerebral blood flow with computed tomography in patients with intracranial masses. Individuals with a mass effect reported reduced cerebral vascular flow on both sides, with a more pronounced decrease on the cystic side..$^{21}$ Based on this finding, we investigated whether fetal CPCs affected the blood flow in the vascular compartment.

Two major factors affecting the vascular blood flow include the resistance of vessel wall and pressure variation reflecting the blood vessel elasticity. The first factor is determined by the functioning of the heart, whereas the second factor depends on the physiological status of the artery and blood need. Both PI and RI reflect the flexibility and resistance of blood vessels in the direction of blood flow. The measurements of PSV, EDV, and S/D ratio are considered significant when the PSV is the primary Doppler parameter in cases of abnormal stenosis. ${ }^{22}$ In our study, both PI and RI values were higher in the CPC group in both MCAs; however, this difference was statistically insignificant. These findings led us to conclude that vessel resistance was slightly higher in the MCAs of the CPC group as compared to the control group. However, the difference was statistically insignificant. In Doppler examination, $\mathrm{S} / \mathrm{D}$ ratio also allows to examine the diastolic blood flow and predict the resistance in the direction of blood flow. ${ }^{23}$ In our study, the MCA PSV values in the CPC group were higher than those in the control group, whereas the EDV values were lower. However, these differences were also not statistically significant. A statistically significant difference was observed only in the S/D ratio. The increased MCA S/D ratio in the CPC group could be attributed to a decrease in the diastolic blood flow. While CPCs did not affect other MCA parameters, these only affected the S/D parameters, which, in turn, could be due to the limited effect on vascular resistance and elasticity. The existence of a statistically insignificant increase in other parameters (particularly in the PI value) may indicate that these cysts also contribute to a slight increase in the vessel resistance. Interestingly, 
these effects were observed on both cystic and contralateral sides. This observation could be explained by the fact that the mass effect of the cyst was observed on the contralateral side as well, or MCA vessels on both sides had an adaptive effect on coordination with each other. However, these findings are in the very early stages and warrant further and a thorough and extensive investigation.

The present study had a few limitations. The main limitation of our study was the limited number of cases. Another limitation was the lack of follow-up of cysts in subsequent weeks. Therefore, we could not confirm whether the effect of cysts on MCA Doppler parameters was temporary. Moreover, this limits our understanding of the predictive effect of MCA Doppler on the regression of CPCs. Future prospective studies are believed to shed more light on the predictive values of MCA Doppler parameters in causing regression of CPCs. Nevertheless, we believe the present study, to the best of our knowledge, to be the first of its kind to evaluate the impact of the mass effect of CPCs on MCA Doppler parameters in the second trimester.

\section{CONCLUSION}

Choroid plexus cysts are sonolucent, fluid-filled structures that generally occur in the lateral ventricles, particularly in the right lateral ventricle.
While CPCs do not affect other MCA Doppler parameters at 18 to 22 weeks of gestation, they increase the S/D ratio significantly. This increase is observed in the MCA of both cystic and contralateral sides.

\section{Source of Finance}

During this study, no financial or spiritual support was received neither from any pharmaceutical company that has a direct connection with the research subject, nor from a company that provides or produces medical instruments and materials which may negatively affect the evaluation process of this study.

\section{Conflict of Interest}

No conflicts of interest between the authors and / or family members of the scientific and medical committee members or members of the potential conflicts of interest, counseling, expertise, working conditions, share holding and similar situations in any firm.

\section{Authorship Contributions}

Idea/Concept: All authors; Design: Cihan İnan, N. Cenk Sayın, Z. Nihal Dolgun, Selen Gürsoy Erzincan, Işıl Uzun, Havva Sütcü; Control/Supervision: All authors; Data Collection and/or Processing: Cihan Inan, N. Cenk Sayın; Analysis and/or Interpretation: Cihan İnan, N. Cenk Sayın, Z. Nihal Dolgun, Füsun Varol; Literature Review: Cihan İnan, N. Cenk Sayın, Z. Nihal Dolgun; Writing the Article: Cihan Inan, N. Cenk Sayın, Z. Nihal Dolgun; Critical Review: All authors; References and Fundings: Cihan İnan.

\section{REFERENCES}

1. Kurjak A, Schulman H, Predanic A, Predanic M, Kupesic S, Zalud I. Fetal choroid plexus vascularization assessed by color flow ultrasonography. J Ultrasound Med. 1994;13(11): 841-4. [Crossref] [PubMed]

2. Zafar HM, Ankola A, Coleman B. Ultrasound pitfalls and artifacts related to six common fetal findings. Ultrasound Q. 2012;28(2):10524. [Crossref] [PubMed]

3. Rochon M, Eddleman K. Controversial ultrasound findings. Obstet Gynecol Clin North Am. 2004;31(1):61-99. [Crossref]

4. Nyberg DA, Souter VL. Sonographic markers of fetal trisomies: second trimester. J Ultrasound Med. 2001;20(6):655-74. [Crossref]

5. Epelman M, Daneman A, Blaser SI, Ortiz-Neira C, Konen O, Jarrín J, et al. Differential diagnosis of intracranial cystic lesions at head US: cor- relation with $\mathrm{CT}$ and $\mathrm{MR}$ imaging. Radiographics. 2006;26(1):173-96. [Crossref] [PubMed]

6. Turner SR, Samei E, Hertzberg BS, DeLong DM, Vargas-Voracek R, Singer A, et al. Sonography of fetal choroid plexus cysts: detection depends on cyst size and gestational age. J Ultrasound Med. 2003;22(11):1219-27. [Crossref] [PubMed]

7. Fong K, Chong K, Toi A, Uster T, Blaser S, Chitayat $D$. Fetal ventriculomegaly secondary to isolated large choroid plexus cysts: prenatal findings and postnatal outcome. Prenat Diagn. 2011;31(4):395-400. [Crossref] [PubMed]

8. Shah N. Prenatal diagnosis of choroid plexus cyst: what next? J Obstet Gynaecol India. 2018;68(5):366-8. [Crossref] [PubMed]

9. Yhoshu E, Mahajan JK, Singh UB. Choroid plexus cysts-antenatal course and postnatal outcome in a tertiary hospital in North India. Childs Nerv Syst. 2018;34(12):2449-53. [Crossref] [PubMed]

10. Bronsteen R, Lee W, Vettraino IM, Huang R, Comstock $\mathrm{CH}$. Second-trimester sonography and trisomy 18: the significance of isolated choroid plexus cysts after an examination that includes the fetal hands. J Ultrasound Med. 2004;23(2):241-5. [Crossref] [PubMed]

11. Danışman N, Ekici E, Vicdan $K$, Gökmen O. [Choroid plexus cysts]. Perinatoloji Dergisi. 1995;3(1):8-12.

12. Irani $S$, Ahmadi F, Javam M, Vosough Taghi Dizaj A, Niknejad F. Outcome of isolated fetal choroid plexus cyst detected in prenatal sonography among infertile patients referred to Royan Institute: a 3-year study. Iran J Reprod Med. 2015;13(9):571-6. [PubMed] [PMC] 
13. Naeini RM, Yoo JH, Hunter JV. Spectrum of choroid plexus lesions in children. AJR Am J Roentgenol. 2009;192(1):32-40. [Crossref] [PubMed]

14. Norton KI, Rai B, Desai H, Brown D, Cohen M. Prevalence of choroid plexus cysts in term and near-term infants with congenital heart disease. AJR Am J Roentgenol. 2011;196(3): W326-9. [Crossref] [PubMed]

15. Chamczuk AJ, Grand W. Endoscopic cauterization of a symptomatic choroid plexus cyst at the foramen of Monro: case report. Neurosurgery. 2010;66(6 Suppl Operative):376-7.

16. Spennato $P$, Chiaramonte $C$, Cicala $D$, Donofrio V, Barbarisi M, Nastro A, et al. Acute triventricular hydrocephalus caused by choroid plexus cysts: a diagnostic and neurosurgical challenge. Neurosurg Focus. 2016;41(5):E9. [Crossref] [PubMed]
17. Tamai S, Hayashi $Y$, Sasagawa $Y$, Oishi M, Nakada M. A case of a mobile choroid plexus cyst presenting with different types of obstructive hydrocephalus. Surg Neurol Int. 2018;9:47. [Crossref] [PubMed] [PMC]

18. Tarzamni MK, Nezami N, Gatreh-Samani F, Vahedinia S, Tarzamni M. Doppler waveform indices of fetal middle cerebral artery in normal 20 to 40 weeks pregnancies. Arch Iran Med. 2009;12(1):29-34. [PubMed]

19. Ursino M, Lodi CA. A simple mathematical model of the interaction between intracranial pressure and cerebral hemodynamics. J Appl Physiol (1985). 1997;82(4):1256-69. [Crossref] [PubMed]

20. Pappalardo EM, Militello M, Rapisarda G, Imbruglia L, Recupero S, Ermito S, et al. Fetal intracranial cysts: prenatal diagnosis and out- come. J Prenat Med. 2009;3(2):28-30 [PubMed] [PMC]

21. Nakayama $Y$, Tanaka A, Kumate S, Yoshinaga S. Cerebral blood flow in normal brain tissue of patients with intracranial tumors. Neurol Med Chir (Tokyo). 1996;36(10):709-14. [Crossref]

22. Oglat $A A$, Matjafri $M Z$, Suardi $N$, Oqlat MA, Abdelrahman MA, Oqlat AA. A review of medical doppler ultrasonography of blood flow in general and especially in common carotid artery. J Med Ultrasound. 2018;26(1):3-13. [Crossref] [PubMed] [PMC]

23. Singh SK, Mishra P. Doppler study of umbilical and fetal middle cerebral artery in severe preeclampsia and intra uterine growth restriction and correlation with perinatal outcome. Int J Reprod Contracept Obstet Gynecol. 2017;6(10):4561-6. [Crossref] 